\title{
Treatment of Adult Metachromatic Leukodystrophy Model Mice Using Intrathecal Administration of Type 9 AAV Vector Encoding Arylsulfatase A
}

Noriko Miyake ( $\nabla$ noriko@nms.ac.jp )

Department of Biochemistry and Molecular Biology, Nippon Medical School

Koichi Miyake

Department of Gene Therapy, Nippon Medical School

Atsushi Sakai

Department of Pharmacology, Nippon Medical School

Motoko Yamamoto

Department of Biochemistry and Molecular Biology, Nippon Medical School

Hidenori Suzuki

Department of Pharmacology, Nippon Medical School

\section{Takashi Shimada}

Department of Biochemistry and Molecular Biology, Nippon Medical School

\section{Research Article}

Keywords: Treatment, adult, metachromatic leukodystrophy, intrathecal, administration, encoding, lysosomal storage disease, vector encoding

Posted Date: July 12th, 2021

DOl: https://doi.org/10.21203/rs.3.rs-687914/v1

License: (c) (i) This work is licensed under a Creative Commons Attribution 4.0 International License. Read Full License 


\section{Abstract}

Metachromatic leukodystrophy (MLD) is a lysosomal storage disease caused by an arylsulfatase A (ASA) deficiency and characterized by severe neurological symptoms resulting from demyelination within the central and peripheral nervous systems. We investigated the feasibility and efficacy of intrathecal administration of a type 9 adeno-associated viral vector encoding ASA (AAV9/ASA) for the treatment of 6-week-old MLD model mice, which are presymptomatic, and 1-year-old mice, which exhibit neurological abnormalities. Immunohistochemical analysis following AAV9/ASA administration showed ASA expression within the brain, with highest activities in the cerebellum and olfactory bulbs. In mice treated at 1 year, alcian blue staining and quantitative analysis revealed significant decreases in stored sulfatide within the hindbrain but not forebrain. Behaviorally, mice treated at 1 year showed no improvement in their ability to traverse narrow balance beams as compared to untreated mice. By contrast, MLD mice treated at 6 weeks showed significant decreases in stored sulfatide throughout the entire brain and improved ability to traverse narrow balance beams. These findings suggest intrathecal administration of an AAV9/ASA vector is a promising approach to treating genetic diseases of the central nervous system, including MLD, though it may be essential to begin therapy before the onset of neurological symptoms.

\section{Introduction}

Metachromatic leukodystrophy (MLD) is an autosomal recessive inherited lysosomal storage disorder caused by a deficiency in the lysosomal enzyme arylsulfatase A (ASA), which catalyzes the degradation of galactosyl-3-sulfate ceramide (sulfatide), a major myelin sphingolipid. ${ }^{1}$ This disease is characterized pathologically by myelin degeneration in both the central and peripheral nervous systems (CNS and PNS). ${ }^{2}$ Clinically, the disease manifests as progressive motor and mental deterioration that is ultimately lethal. ${ }^{3}$ MLD is classified into three or four different forms based on the age of its onset. ${ }^{4,5}$ Late infantile is the most frequently observed and severe type. ${ }^{6}$ These patients usually develop neurological symptoms that include gait disturbance and loss of speech at around 2 years of age and die within a few years after appearance of the first symptoms. ${ }^{1}$ Although enzyme replacement therapy using human ASA has been tried, this approach does not effectively relieve the neurological symptoms (NCT00681811; http://www.clinicaltrials.gov). A novel therapy that can stop or reverse the progression of this neurological disorder is therefore needed.

Gene therapy is one of the potentially effective strategies under consideration for use in the treatment of CNS disorders, and several gene therapy protocols for treating MLD have been proposed. ${ }^{7}$ However, the blood brain barrier (BBB), which limits the delivery of systemically administered therapeutic molecules to the brain, is a major obstacle to successful treatment of any neurological disorder affecting the CNS..$^{8,9}$ Consequently, how to deliver a therapeutic gene across the BBB and then how to distribute it to the entire brain are crucial challenges that must be overcome. One simple strategy is direct injection of viral vectors into the brain; ${ }^{10,11}$ another is hematopoietic stem cell gene therapy. ${ }^{12,13}$ We recently succeeded in treating neonatal ASA knockout MLD model mice through systemic gene delivery of a single-strand type 9 adeno- 
associated viral vector encoding human ASA (AAV9/ASA). ${ }^{14}$ Unfortunately, however, intravenous systemic gene delivery is much less effective in adult mice than neonatal mice. We also previously showed in ASA knockout mice that intrathecal injection of a type 1 AAV vector encoding ASA into a single site within the cisternal space results in widespread gene delivery to the brain and dorsal root ganglia (DRG). ${ }^{15}$ Other groups have also demonstrated the utility of intrathecal delivery of type 9 AAV for transduction within the CNS, ${ }^{16-18}$ and this strategy has been used to treat the lysosomal storage diseases MPS I and Pompe disease. ${ }^{19,20}$

In the present study, we used a type 9 AAV vector previously shown to efficiently transduce the $\mathrm{CNS}^{21-23}$ and tested the feasibility and potential efficacy of intrathecal administration of AAV9/ASA for the treatment of MLD in adult mice. In addition, to determine whether overt neurological symptoms could be reversed by the gene therapy, we also assessed the effect of AAV9/ASA in 1-year-old MLD mice, which already exhibited the neurological symptoms of the disease. Since the AAV gene therapy for MLD has yet to be tested intrathecally and that human trials have been limited to ex vivo lentiviral mediated hematopoietic stem cells gene therapy, ${ }^{12,13}$ it seems important to examine the effect of these intrathecal administration of AAV9/ASA for the treatment of MLD.

\section{Materials And Methods}

\section{Animals}

ASA knockout (MLD) mice were a gift from the laboratory of Dr. V. Gieselmann. ${ }^{32}$ These mice have been extensively characterized and are widely used as a mouse model of MLD. ${ }^{33,34}$ Mice were housed under a $12 \mathrm{~h}$ light-dark cycle and had ad libitum access to food and water. All animal experiments were performed in accordance with animal welfare laws, complied with ARRIVE guidelines, and the regulations of the Ethics Committee of Nippon Medical School. All experimental protocols were approved by the responsible committees of Nippon Medical School.

\section{Generation of $A A V$ vectors}

The recombinant AAV vector plasmid pAAV/GFP, which contains the enhanced GFP gene driven by the CAG promoter, was described previously. ${ }^{35}$ The recombinant AAV vector plasmid pAAV/ASA, which contains CDNA encoding flag-tagged ASA driven by the CAG promoter as the first gene and GFP CDNA driven by the B19 promoter as the second gene, has also been described previously. ${ }^{15}$ AAV serotype 9 packaging plasmids (p5E18VD2/9) were kindly provided by Dr. J. Wilson. ${ }^{36}$ Recombinant AAV vectors were generated using a three-plasmid co-transfection system (vector plasmid, packaging plasmid and helper plasmid (pHelper; Stratagene, La Jolla, CA)) and then purified using ammonium sulfate precipitation and iodixanol continuous gradient centrifugation, as described previously. ${ }^{14,37}$ The titer of each AAV vector was determined using real-time PCR (7500 Fast, Applied Biosystems, Tokyo, Japan). The approximate titer of the final preparation of each AAV vector was $4.0 \times 10^{13}$ vector genomes $(\mathrm{vg}) / \mathrm{ml}$. 


\section{Intrathecal injection}

Six-week-old or 1-year-old MLD mice were deeply anesthetized using pentobarbital, placed in a stereotactic frame and flexed in a prone position, after which the back of the neck was cleaned with povidone-iodine. A skin incision was then made at the back of the head for intrathecal injection into the suboccipital cisternal space. The dura mater was exposed between the occipital bone and cervical vertebrae, and a needle was inserted into the cistern via a suboccipital puncture. Ten microliters of vector solution containing $4.0 \times 10^{11} \mathrm{vg}$ AAV particles were delivered over a period of 1 min using a Hamilton syringe with a 33-g bevel-tipped needle (Hamilton, Reno, NV). ${ }^{18,38}$ These injection steps were performed manually.

\section{Immunohistochemical analysis}

Immunohistochemical analysis of neuronal cells was performed as described previously. ${ }^{24}$ Mice were anesthetized and perfused with phosphate-buffered saline (PBS). The brains and spinal cords were then dissected out, fixed in $4 \%$ paraformaldehyde overnight at $4^{\circ} \mathrm{C}$, and immersed in PBS containing $20 \%$ sucrose. Thereafter, the specimens were placed in OCT compound (Tissue-Tek, Tokyo, Japan), and tissue sections were cut to a thickness of $20 \mu \mathrm{m}$ and mounted on glass slides. The sections were then immunostained for ASA, neurons, cholinergic neurons, astrocytes and GFP by incubation for $16 \mathrm{~h}$ at $4^{\circ} \mathrm{C}$ with goat anti-ASA (AF2485; $5 \mu \mathrm{g} / \mathrm{ml}$, R\&D, Minneapolis, MN), mouse anti-NeuN (MAB377; 1:500, Millipore, Billerica, MA), rabbit anti-calbindin D-28K (AB1778; 1:1000, Millipore), mouse anti-parvalbumin (P3088; 1:2000, Sigma-Aldrich, St Louis, MO), goat anti-choline acetyltransferase (ChAT) (AB144P; 1:100, Millipore), mouse anti-GFAP (G3893; 1:500, Sigma-Aldrich), rabbit anti-lba1 (019-19741; 1:500, FUJIFILM, Osaka, Japan), and rabbit anti-GFP (A21311; 1:1000, Invitrogen, Life Technologies Corp., Carlsbad, CA) or rat anti-GFP (04404-84; 1:1000, Nacalai tesque, Kyoto, Japan) antibodies. After washing the sections three times with PBS, they were stained with Alexa Fluor 568-conjugated anti-mouse, anti-rabbit or antigoat IgG (1:500, Invitrogen) or with Alexa Fluor 488-conjugated anti-rabbit IgG or anti-rat IgG (1:500, Invitrogen). Finally, the sections were examined under a BX60 microscope (Olympus, Tokyo, Japan).

\section{Determination of ASA concentrations}

Each organ of interest was homogenized in pure water, after which the human ASA (ASA) concentration was determined using an indirect sandwich enzyme-linked immune sorbent assay (ELISA), as described previously. ${ }^{15}$ Human ASA does not cross-react with murine ASA. ${ }^{39}$

\section{Droplet digital PCR (ddPCR) analysis for detection of vector genome}

Total DNA was isolated from AAV/ASA-transduced mouse tissues using a DNeasy Tissue Kit (QIAGEN, Hilden, Germany), after which the DNA was subjected to droplet digital PCR to detect the copy number of the AAV vector. The mouse haploid genome single-copy gene transferrin receptor (TFRC) (4458366: Thermo Fisher scientific) was selected as an internal control. Each PCR reaction was run in a $20-\mu \mathrm{L}$ reaction mixture containing $10 \mu \mathrm{L}$ of ddPCR supermix for probes (Bio-Rad), $900 \mathrm{nM}$ primers, $250 \mathrm{nM}$ 
probe and $4 \mu \mathrm{L}$ of template DNA. Droplets were generated on a Bio-Rad QX-200 following the manufacturer's instructions. Samples were transferred to a 96 well-plate and thermal cycled to the endpoint (T100 Thermal Cycler; Bio-Rad) using a standard protocol: initial denaturation at $95^{\circ} \mathrm{C}$ for 10 min followed by 40 cycles of melting at $94^{\circ} \mathrm{C}$ for $30 \mathrm{~s}$ and annealing/elongation at $55^{\circ} \mathrm{C}$ for 1 min before droplet enzyme deactivation by incubation for $10 \mathrm{~min}$ at $98^{\circ} \mathrm{C}$. The PCR plate was subsequently scanned using a QX200 droplet reader (BIO-RAD), and the data were analyzed using QuantaSoft software (BIORAD). For 2D ddPCR, two sets of primers and probes labeled with FAM and VIC, respectively, were added to each reaction mixture, and ddPCR was performed in the same tube. The following primers and probe were used for ddPCR: 5'- TACATCAAGTGTATCATATGCCAA -3' (CAG probe), 5'-

CAATGGGTGGAGTATTTACG-3' (forward primer), and 5'- GGTCATGTACTGGGCATAAT-3' (reverse primer). TaqMan $^{\text {TM }}$ copy number reference assay (mouse, $T f r c$ ) was used as an internal control. The vector genome were showed CAG/Tfrc ratios.

\section{Alcian blue staining and analysis of Sulf and GalC}

To detect the accumulation of sulfatides, brain and spinal cord sections were stained with alcian blue (Sigma-Aldrich) as described previously. ${ }^{40}$ Sulfatide levels were determined as follows. The excised brains, separated into two segments (forebrain and hindbrain comprising the brainstem and cerebellum), and spinal cords were homogenized in pure water, after which the homogenates were centrifuged at $15,000 \mathrm{rpm}$ for $10 \mathrm{~min}$ at $4^{\circ} \mathrm{C}$. The supernatants were then collected for determination of the protein content using a Bio-Rad DC Assay (Bio-Rad, Hercules, CA) and sulfatide content using thin-layer chromatography. Crude lipids were extracted from the tissue homogenates using the Folch method. ${ }^{41}$ The levels of sulfatide and galactosylceramide present were determined by densitometric scanning using a DT-20 MCP (Atto, Tokyo, Japan), as described previously. ${ }^{10,24}$ Amounts were quantitatively determined with densitometric scanning using CS Analyzer 3 (ATTO, Tokyo, Japan). Data are expressed as the mean \pm standard error (SE) of Sulf/GalC ratios.

\section{Behavior test}

Motor coordination and balance were assessed in the mice by measuring their ability to traverse a graded series of narrow beams to reach an enclosed safety platform as described previously. ${ }^{42}$ The beams consisted of long $(1 \mathrm{~m})$ stainless steel rods, starting at a cross-sectional diameter of $20 \mathrm{~mm}$ (O'HARA \& Co., Ltd., Tokyo Japan). The beams were placed horizontally, $50 \mathrm{~cm}$ above the bench surface, with one end mounted on a narrow support and the other end attached to an enclosed box $\left(20 \mathrm{~cm}^{2}\right)$, into which a mouse could escape. The mice were trained to traverse the beam to the enclosed box. The time required to traverse each beam ("latency") and the number of times the hind feet slipped off a beam ("slips") were recorded for each trial. Analysis of each measure was based on the mean scores from three trials.

\section{Statistical analysis}


Results are expressed as means \pm SE of duplicate or more data obtained from three or more independent experiments. Data were analyzed using two-tailed Student's t-tests. Values of $p<0.05$ were considered significant.

\section{Results}

\section{Intrathecally injected AAV9/GFP is efficiently transduced into the cerebellum, brainstem, spinal cord and DRG}

We first analyzed the transduction efficiency and distribution of an AAV9 vector encoding green fluorescent protein (AAV9/GFP: $4.0 \times 10^{11} \mathrm{vg}$ ) in 1-year-old and 6-week-old MLD mice following intrathecal injection via a suboccipital puncture. The distribution of AAV9/GFP in the brain, spinal cord and DRG was then determined 3 or 13.5 months after the injection through immunohistochemical detection of GFP. Efficient GFP expression was detected in the midbrain, brainstem, cerebellum (Figure 1a, c) and both the cervical and lumbar spinal cord (Figure 1b). In addition, a large number of nerve fibers in the dorsal spinal cord and numerous neuronal cell bodies in the DRG were also transduced (Figure 1b). These results may indicate that DRG neurons are efficiently transduced by intrathecal injection of AAV9 vector and that GFP is transported via ascending fibers in the dorsal spinal cord.

\section{Efficient ASA expression in the cerebellum and spinal cord}

We next treated MLD mice using an AAV9 vector encoding ASA together with GFP (AAV9/ASA). These vectors were intrathecally injected into 6-week-old MLD mice, which were presymptomatic, and 1-year-old MLD model mice, which already showed neurological symptoms. 13.5 months or 3 months after injection, when both groups of mice were around 15 months old, we used immunostaining to assess expression of ASA and GFP. Figure 2 shows the results of intrathecal injection of AAV9/ASA into 1-yearold MLD mice. Efficient ASA expression was detected throughout the GFP-positive areas, with the cerebellum and spinal cord showing the highest activities (Figure 2b). Nearly the same pattern of ASA expression was observed after intrathecal injection into 6-week-old MLD mice (data not shown). For MLD mice treated at 1 year, we also used ELISAs to detect levels of ASA (Figure 2c) and the vector genome in the forebrain, hindbrain, spinal cord and liver (Figure 2d). ASA protein and the vector genome were detected in hindbrain $(4.9 \pm 2.7 \mathrm{ng} / \mathrm{mg}$ and $0.2 \pm 0.1 \mathrm{copy} /$ cell $)$ and spinal cord $(3.5 \pm 2.2 \mathrm{ng} / \mathrm{mg}$ and 1.2 \pm 0.1 copy /cell) but not in the forebrain. ASA protein and the vector genome were also detected in the liver $(1.2 \pm 0.1 \mathrm{ng} / \mathrm{mg}$ and $1.5 \pm 1.0 \mathrm{copy} /$ cell $)$.

Further immunostaining for the appropriate markers showed that GFP-positive cells included NeuNpositive neurons (Figure 3a), GFAP-positive glial cells (Figure 3B), calbindin-positive Purkinje cells (Figure 3c), parvalbumin-positive basket-cells (Figure 3d) in the brain or choline acetyl transferase (ChAT)positive motor neurons (Figure 3f) and NeuN-positive neurons (Figure 3g) in the cervical spinal cord, especially the ventral horn. On the other hand GFP-positive cells did not include Iba1-positive microglia (Figure $3 e$ ) in the brain. Within the brains of treated MLD mice, $72.3 \pm 5.7 \%$ of GFP-positive cells were 
neurons and $27.3 \pm 5.8 \%$ of GFP-positive cells were astrocytes in mice treated at 1 year, while $87.3 \pm 5.3$ $\%$ of GFP-positive cells were neurons and $12 \pm 5 \%$ of GFP-positive cells were astrocytes in mice treated at 6 weeks. We detected no GFP-positive oligodendrocytes. Thus, we did not find significant difference between 1-year-old and 6-week-old treated MLD mice.

\section{Reductions in sulfatide storage and Sulf/GalC ratios in the CNS}

To analyze the potential therapeutic effect of intrathecal injection of AAV9/ASA into MLD mice, we used alcian blue staining to detect the presence of sulfatides. Sulfatide accumulation within cells was readily detected in 15-month-old untreated MLD mice. Sulfatide was especially detectable in the white matter, forebrain (including the corpus callosum and hippocampal fimbria), hindbrain (cerebellar nucleus and vestibular nucleus) and ventral horn of the spinal cord (Figure 4a). Notably, MLD mice treated at 1 year or 6 weeks showed markedly less sulfatide accumulation within the brain and spinal cord than did agematched untreated MLD mice (Figure 4a).

We also used thin-layer chromatography to quantitatively analyze the abundance of sulfatide in lipid extracts. Sulfatide storage in the brain was evaluated based on the ratio of sulfatide (Sulf) to galactosylceramide (GalC), which is a major component of the myelin sheath in oligodendrocytes. We found that as compared to untreated MLD mice, Sulf/GalC ratios were significantly reduced in the forebrain of MLD mice treated at 6 weeks $(0.66 \pm 0.05$ vs. $0.87 \pm 0.03, p<0.05)$, in the hindbrain of mice treated at 1 year or 6 weeks $(0.55 \pm 0.03$ or $0.45 \pm 0.06$ vs. $0.67 \pm 0.04, p<0.05)$ and in the cervical spinal cord of mice treated at 1 year or 6 weeks $(0.25 \pm 0.01$ or $0.25 \pm 0.02$ vs. $0.39 \pm 0.02, p<0.05$ (Figure 4b). In addition, alcianophilic material was almost entirely absent from the spinal cord, which is nearly identical to what is observed in wild type mice.

Finally, to assess the effect of AAV9/ASA treatment on microglial activation, we evaluated staining with anti-lba1 antibody (Figure 5a). There was reduction of activated microglia in the both the cerebellum and spinal cord of AAV9/ASA-treated MLD mice compare to untreated mice (cerebellum: treated at 6 weeks, $15.5 \pm 1.85 \%$; treated at 1 year, $17.0 \pm 2.6 \%$; untreated, $41.1 \pm 7.5 \%$; spinal cord: treated at 6 weeks, $21.57 \pm 3.3 \%$; treated at 1 year, $12.3 \pm 0.7 \%$; untreated, $60.7 \pm 7.9 \%$ ) (Figure $5 b$ ).

\section{MLD mice exhibit significant improvement in a behavioral test when treated at 6 weeks of age but not at 1 year}

To evaluate the effect of intrathecal AAV9/ASA injection on the neurological symptoms in MLD mice, we compared the abilities of treated and untreated mice to perform a behavioral (balance beam) test as previously described. ${ }^{24}$ We found that MLD mice treated at 6 weeks performed significantly better than the untreated mice (latency: $9.4 \pm 0.9$ s vs. $18.9 \pm 2.8$ s, $p<0.03$; number of slips: $6.2 \pm 1.3$ vs. $11.2 \pm 2.0$, $p<0.05$ ). Although, the latency of MLD mice treated at 6 weeks was nearly same as that of wild-type mice, the treated MLD mice still exhibited a significantly greater number of slips than wild-type mice. Moreover, MLD mice treated at 1 year showed no significant improvement as compared to the untreated MLD mice (latency: 15.5 \pm 1.7 s, number of slips: $12.6 \pm 3.4$ ) (Figure 6). 


\section{Discussion}

We previously reported that after intravenous systemic injection of AAV, the vectors were able to pass through the BBB of neonatal mice and efficiently transduce the CNS. ${ }^{25}$ However, unfortunately, systemic administration of AAV vectors is less effective in adult mice than neonatal mice. To address that problem, we tested the utility of intrathecal injection of AAV in adult mice. Following intrathecal injection of an AAV9/GFP vector, GFP expression was seen in the hindbrain and cerebellum. In addition, a large number of nerve fibers in the dorsal spinal cord and numerous neuronal cell bodies in the DRG were also efficiently transduced. Although more invasive than an intravenous injection, intrathecal injection may enable use of lower vector doses than are used for intravenous administration. Moreover, with intrathecal injection there is less chance of either germline transduction or generation of neutralizing antibodies than with intravenous administration. ${ }^{16}$ Thus, intrathecal injection of AAV vectors could prove highly useful for efficient and long-term expression of desired genes in the CNS and spinal cord and could be an effective means of treating genetic neurological diseases.

In the present study, we clearly demonstrated that intrathecal infusion of AAV9/ASA into adult MLD mice resulted in efficient gene delivery to the cerebellum, spinal cord and DRG. Overall, however, GFP and ASA expression were detected in only a limited area of the brain following intrathecal injection of AAV9 vector. Because MLD is a whole brain disease, this limited ASA expression may be not so sufficient to eliminate the MLD disease phenotype. In addition, because MLD is a demyelination disease, we assessed ASA protein expression in myelin forming cells. Glial cells positive for both GFAP and ASA were detected (Figure 3b), but not microglia positive for both Iba1 and ASA (Figure 3e). Nevertheless, inhibition of sulfatide accumulation and significantly improved performance of a behavioral (balance beam) test were observed after treatment of 6-week-old mice. One potential explanation for this beneficial effect is the occurrence of "cross-correction," whereby transduced cells corrected by the gene therapy secrete the transduced lysosomal enzyme, which is then be taken up by neighboring cells via the M6P (mannose 6phosphate) receptor. ${ }^{26}$ These successful corrections of biochemical and neurological abnormalities suggest that AAV9-mediated intrathecal gene therapy is a promising approach to treating lysosomal storage diseases with neurological involvement.

In this study, the area transduced through intrathecal injection was limited to the hindbrain and cerebellum. This is in contrast to the findings of two studies that reported broad gene transfer throughout the brain, including the forebrain, following intrathecal injection. ${ }^{22,27}$ We speculate that the reasons for this discrepancy may involve the structure of the AAV vector (single stranded vs. self-complementary, WPRE (-) vs. WPRE (+)), the injection site (cistern vs. lumbar region), and/or the promoter used (CAG vs CBA promoter). In addition, when Meyer et al. described transduction of the entire CNS in adult nonhuman primates through intrathecal delivery of scAAV9-CBA-GFP, they reported that transduction was more efficient when primates were kept in the Trendelenburg position following administration of the AAV vector. ${ }^{28}$ Along the same lines, Bay et al. reported holding mice in a tilted $30^{\circ}$ head-down position for 6 min as part of the intrathecal injection protocol. Thus, in addition to the route of vector delivery, the 
position of the animal and the impact of gravity may also be an important factor significantly affecting the distribution of vector. ${ }^{23}$

Moreover, because we performed the injection manually, it is possible that variation in the needle depth during the injection resulted in some vector being injected intraparenchymally. This would explain the strong bias for gene expression in brain regions immediately beneath the cisterna magna. It might be helpful to examine the motor cortex in these mice, as specific transduction of cortical motor neurons can occur as a result of retrograde transport following intraparenchymal injection of AAV9 in the hindbrain (beneath the cisterna magna).

To test whether overt neurological symptoms can be reversed by gene therapy, we intrathecally administered AAV9/ASA to 1-year-old MLD mice, which already exhibit neurological symptoms of the disease. Efficient ASA expression and a decrease in sulfatides were detected in the hindbrain and spinal cord of mice treated at 1 year. Behaviorally, however, the treated mice showed no significant improvement over untreated control MLD mice, even though the transduction efficiency and ASA expression did not differ between mice treated at 1 year and 6 weeks. We speculate that significant improvement was observed in mice treated at 6 weeks but not 1 year because ASA expression after intrathecal injection of AAV/ASA inhibited further accumulation of sulfatide, but did not reduce the amount of sulfatide that accumulated prior to treatment. Alternatively, or in addition, it may be that irreversible histological changes induced by MLD had already occurred by 1 year of age. In addition, although the sulfatide content was lower in treated than untreated MLD model mice, the treatment-induced reduction of sulfatide was limited. The therapeutic effect of a higher dose of vector should therefore evaluated. That said, our findings suggest that for successful treatment of MLD, it is essential to start gene therapy before the onset of neurological symptoms.

Currently ongoing gene therapy for MLD patients entails bone marrow transplantation of ASA-expressing stem cells transduced with a lentiviral vector. ${ }^{13}$ However, it is still unclear whether the transplanted cells pass through the BBB and reverse the neurological disorder in MLD patients. Sevin et al. demonstrated a small trial evaluating direct injection of AAVrh.10-ARSA vector in patients with MLD (NCT01801709). However, this study was terminated due to lack of effectiveness. ${ }^{29}$ By contrast, intrathecally injected AAV vector is clearly able to transduce the CNS and mediate ASA expression in the brain. This strategy may thus be an effective means of treating neurological disorders. At present, however, only limited CNS transduction has been achieved with this approach. It was recently reported that a combination gene therapy entailing direct injection into the brain together with intrathecal injection was more effective than either single treatment alone. The efficacy of such combination therapy has been demonstrated in the Sanfilippo syndrome type B model mouse ${ }^{30}$ and in cases of canine mucopolysaccharidosis VII. ${ }^{31}$ In MLD, sulfatide accumulation is detected not only in the CNS, but also in the heart, liver, kidney and other organs. Systemic intravenous injection of AAV9/ASA would likely decrease sulfatide accumulation in those organs. We previously reported that intraventricular injection of a type 1 self-complementary (sc) AAV vector encoding ASA suppressed sulfatide accumulation in MLD mice. Noting that Snyder et al. reported superior transduction properties of AAV6 and 9 for intrathecal injection as compared to 
AAV1, ${ }^{18}$ we used an AAV9 vector in the present study. However, we used a single stranded AAV9 vector, which may have reduced the efficacy after cisterna magna delivery. Combination gene therapy entailing both intraventricular and intrathecal injection of scAAV/ASA may be a more effective strategy for treating 1-year-old MLD mice and could be potentially useful for treating MLD patients.

We used ELISAs to assess delivery of ASA and the vector genome to the brain, spinal cord, and liver of intrathecally injected MLD mice. ASA protein and the vector genome were detected not only in the brain and spinal cord, but also in the liver, which indicates that intrathecally injected AAV/ASA enters the bloodstream and travels throughout the circulation, reaching the liver. It will therefore be important to analyze vector distribution in various tissues; and to address concern about potential germline transmission, evaluation of gene transduction of the testis or ovary will be particularly important.

In summary, we have succeeded in treating 6-week-old MLD model mice through intrathecal injection of AAV9/ASA. However, significant improvement of motor behavior was not observed following treatment of 1-year-old MLD mice. Effective treatment of MLD may require that gene therapy be started before the onset of neurological symptoms, or perhaps using a strategy that achieves more widespread transduction within the brain. Combined intrathecal and intraventricular injection of AAV vectors may be such an approach to treating MLD mice already exhibiting neurological symptoms.

\section{Declarations}

\section{ACKNOWLEDGMENTS}

We thank Dr. Volkmar Gieselmann at Rheinische Friedrich-Wilhelms-Universität for kindly providing MLD model mice and Dr. James Wilson at the University of Pennsylvania for providing AAV packaging plasmids. This work was supported in part by grants from the Ministry of Health and Welfare of Japan and the Ministry of Education, Science and Culture of Japan. This work was supported by JSPS KAKENHI Grant Number 15K09604 and 18K07859.

\section{AUTHORS' CONTRIBUTIONS}

N.M., K.M., and T.S. conceived, designed, and administered the study, and all other authors assigned in its design. N.M., S.A., and Y.M. performed experiments and analyzed data. K.M., H.S., and T.S. validated the experimental data. N.M. wrote the manuscript with review comments from all authors. N.M. and K.M. edited the paper. All authors read and approved the final draft of the manuscript.

\section{CONFLICTS OF INTEREST}

The authors have no conflicts of interest to declare.

\section{References}


1. von Figura, K., Gieselmann, V. \& Jaeken, J. Metachromatic leukodystrophy. The Metabolic and Molecular Bases of Inherited Disease. 3695-3724 (McGraw-Hill, 2001).

2. Gieselmann, V. Metachromatic leukodystrophy: recent research developments. J Child Neurol 18, 591594, doi:10.1177/08830738030180090301 (2003).

3. Parikh, S. et al. A clinical approach to the diagnosis of patients with leukodystrophies and genetic leukoencephelopathies. Mol Genet Metab 114, 501-515, doi:10.1016/j.ymgme.2014.12.434 (2015).

4. Sevin, C., Aubourg, P. \& Cartier, N. Enzyme, cell and gene-based therapies for metachromatic leukodystrophy. J. Inherit. Metab. Dis. 30, 175-183, doi:10.1007/s10545-007-0540-z (2007).

5. Groeschel, S. et al. Metachromatic leukodystrophy: natural course of cerebral MRI changes in relation to clinical course. J Inherit Metab Dis 34, 1095-1102, doi:10.1007/s10545-011-9361-1 (2011).

6. Gieselmann, V. \& Krageloh-Mann, I. Metachromatic leukodystrophy--an update. Neuropediatrics 41, 1-6, doi:10.1055/s-0030-1253412 (2010).

7. Rosenberg, J. B., Kaminsky, S. M., Aubourg, P., Crystal, R. G. \& Sondhi, D. Gene therapy for metachromatic leukodystrophy. J. Neurosci. Res. 94, 1169-1179, doi:10.1002/jnr.23792 (2016).

8. Abbott, N. J. Blood-brain barrier structure and function and the challenges for CNS drug delivery. J Inherit Metab Dis 36, 437-449, doi:10.1007/s10545-013-9608-0 (2013).

9. Muldoon, L. L. et al. Immunologic privilege in the central nervous system and the blood-brain barrier. J Cereb Blood Flow Metab 33, 13-21, doi:10.1038/jcbfm.2012.153 (2013).

10. Kurai, T. et al. AAV1 mediated co-expression of formylglycine-generating enzyme and arylsulfatase a efficiently corrects sulfatide storage in a mouse model of metachromatic leukodystrophy. Mol. Ther. 15, 38-43, doi:10.1038/sj.mt.6300012 (2007).

11. Piguet, F. et al. Correction of brain oligodendrocytes by AAVrh.10 intracerebral gene therapy in metachromatic leukodystrophy mice. Hum. Gene Ther. 23, 903-914, doi:10.1089/hum.2012.015 (2012).

12. Biffi, A. et al. Lentiviral hematopoietic stem cell gene therapy benefits metachromatic leukodystrophy. Science 341, 1233158, doi:10.1126/science.1233158 (2013).

13. Sessa, M. et al. Lentiviral haemopoietic stem-cell gene therapy in early-onset metachromatic leukodystrophy: an ad-hoc analysis of a non-randomised, open-label, phase 1/2 trial. The Lancet 388, 476-487, doi:10.1016/s0140-6736(16)30374-9 (2016).

14. Miyake, N., Miyake, K., Asakawa, N., Yamamoto, M. \& Shimada, T. Long-term correction of biochemical and neurological abnormalities in MLD mice model by neonatal systemic injection of an AAV serotype 9 vector. Gene Ther 21, 427-433, doi:10.1038/gt.2014.17 (2014). 
15. Iwamoto, N. et al. Global diffuse distribution in the brain and efficient gene delivery to the dorsal root ganglia by intrathecal injection of adeno-associated viral vector serotype 1. J Gene Med 11, 498-505, doi:10.1002/jgm.1325 (2009).

16. Gray, S. J., Nagabhushan Kalburgi, S., McCown, T. J. \& Jude Samulski, R. Global CNS gene delivery and evasion of anti-AAV-neutralizing antibodies by intrathecal AAV administration in non-human primates. Gene Ther. 20, 450-459, doi:10.1038/gt.2012.101 (2013).

17. Hirai, $T$. et al. Intrathecal shRNA-AAV9 inhibits target protein expression in the spinal cord and dorsal root ganglia of adult mice. Hum Gene Ther Methods 23, 119-127, doi:10.1089/hgtb.2012.035 (2012).

18. Snyder, B. R. et al. Comparison of adeno-associated viral vector serotypes for spinal cord and motor neuron gene delivery. Hum. Gene Ther. 22, 1129-1135, doi:10.1089/hum.2011.008 (2011).

19. Hordeaux, J. et al. Long-term neurologic and cardiac correction by intrathecal gene therapy in Pompe disease. Acta Neuropathol Commun 5, 66, doi:10.1186/s40478-017-0464-2 (2017).

20. Storek, B. et al. Intrathecal long-term gene expression by self-complementary adeno-associated virus type 1 suitable for chronic pain studies in rats. Mol. Pain 2, 4, doi:10.1186/1744-8069-2-4 (2006).

21. Hinderer, C. et al. Widespread gene transfer in the central nervous system of cynomolgus macaques following delivery of AAV9 into the cisterna magna. Mol Ther Methods Clin Dev 1, 14051, doi:10.1038/mtm.2014.51 (2014).

22. Bailey, R. M., Rozenberg, A. \& Gray, S. J. Comparison of high-dose intracisterna magna and lumbar puncture intrathecal delivery of AAV9 in mice to treat neuropathies. Brain Res. 1739, 146832, doi:10.1016/j.brainres.2020.146832 (2020).

23. Bey, K. et al. Efficient CNS targeting in adult mice by intrathecal infusion of single-stranded AAV9-GFP for gene therapy of neurological disorders. Gene Ther. 24, 325-332, doi:10.1038/gt.2017.18 (2017).

24. Miyake, N., Miyake, K., Karlsson, S. \& Shimada, T. Successful treatment of metachromatic leukodystrophy using bone marrow transplantation of HoxB4 overexpressing cells. Mol Ther 18, 13731378, doi:10.1038/mt.2010.74 (2010).

25. Miyake, N., Miyake, K., Yamamoto, M., Hirai, Y. \& Shimada, T. Global gene transfer into the CNS across the BBB after neonatal systemic delivery of single-stranded AAV vectors. Brain Res 1389, 19-26, doi:10.1016/j.brainres.2011.03.014 (2011).

26. Byrne, B. J., Falk, D. J., Clement, N. \& Mah, C. S. Gene therapy approaches for lysosomal storage disease: next-generation treatment. Hum. Gene Ther. 23, 808-815, doi:10.1089/hum.2012.140 (2012).

27. Schuster, D. J. et al. Biodistribution of adeno-associated virus serotype 9 (AAV9) vector after intrathecal and intravenous delivery in mouse. Front. Neuroanat. 8, 42, doi:10.3389/fnana.2014.00042 
(2014).

28. Meyer, K. et al. Improving single injection CSF delivery of AAV9-mediated gene therapy for SMA: a dose-response study in mice and nonhuman primates. Mol. Ther. 23, 477-487, doi:10.1038/mt.2014.210 (2015).

29. Sevin, C. et al. Intracerebral gene therapy in children with metachromatic leukodystrophy: Results of a phase I/II trial. Mol. Genet. Metab. 123, S129, doi:doi: 10.1016/j.ymgme.2017.12.352 (2018).

30. Fu, H. et al. Significantly increased lifespan and improved behavioral performances by rAAV gene delivery in adult mucopolysaccharidosis IIIB mice. Gene Ther. 14, 1065-1077, doi:10.1038/sj.gt.3302961 (2007).

31. Gurda, B. L. et al. Evaluation of AAV-mediated Gene Therapy for Central Nervous System Disease in Canine Mucopolysaccharidosis VII. Mol. Ther. 24, 206-216, doi:10.1038/mt.2015.189 (2016).

32. Hess, B. et al. Phenotype of arylsulfatase A-deficient mice: relationship to human metachromatic leukodystrophy. Proc Natl Acad Sci U S A 93, 14821-14826 (1996).

33. D'Hooge, R., Coenen, R., Gieselmann, V., Lullmann-Rauch, R. \& De Deyn, P. P. Decline in brainstem auditory-evoked potentials coincides with loss of spiral ganglion cells in arylsulfatase A-deficient mice. Brain Res 847, 352-356 (1999).

34. Wittke, D., Hartmann, D., Gieselmann, V. \& Lullmann-Rauch, R. Lysosomal sulfatide storage in the brain of arylsulfatase A-deficient mice: cellular alterations and topographic distribution. Acta Neuropathol. 108, 261-271, doi:10.1007/s00401-004-0883-6 (2004).

35. Noro, T. et al. Adeno-associated viral vector-mediated expression of endostatin inhibits tumor growth and metastasis in an orthotropic pancreatic cancer model in hamsters. Cancer Res 64, 7486-7490, doi:10.1158/0008-5472.CAN-03-1296 (2004).

36. Gao, G. P. et al. Novel adeno-associated viruses from rhesus monkeys as vectors for human gene therapy. Proc Natl Acad Sci U S A 99, 11854-11859, doi:10.1073/pnas.182412299 (2002).

37. Miyake, K., Miyake, N., Yamazaki, Y., Shimada, T. \& Hirai, Y. Serotype-independent method of recombinant adeno-associated virus (AAV) vector production and purification. J Nippon Med Sch 79, 394402 (2012).

38. Fu, H. et al. Self-complementary adeno-associated virus serotype 2 vector: global distribution and broad dispersion of AAV-mediated transgene expression in mouse brain. Mol Ther 8, 911-917 (2003).

39. Yamazaki, Y., Hirai, Y., Miyake, K. \& Shimada, T. Targeted gene transfer into ependymal cells through intraventricular injection of AAV1 vector and long-term enzyme replacement via the CSF. Scientific reports 4, 5506, doi:10.1038/srep05506 (2014). 
40. Scott, J. E. \& Dorling, J. Differential staining of acid glycosaminoglycans (mucopolysaccharides) by alcian blue in salt solutions. Histochemie 5, 221-233 (1965).

41. Folch, J., Lees, M. \& Sloane Stanley, G. H. A simple method for the isolation and purification of total lipides from animal tissues. J Biol Chem 226, 497-509 (1957).

42. Carter, R. J. et al. Characterization of progressive motor deficits in mice transgenic for the human Huntington's disease mutation. J Neurosci 19, 3248-3257 (1999).

\section{Figures}

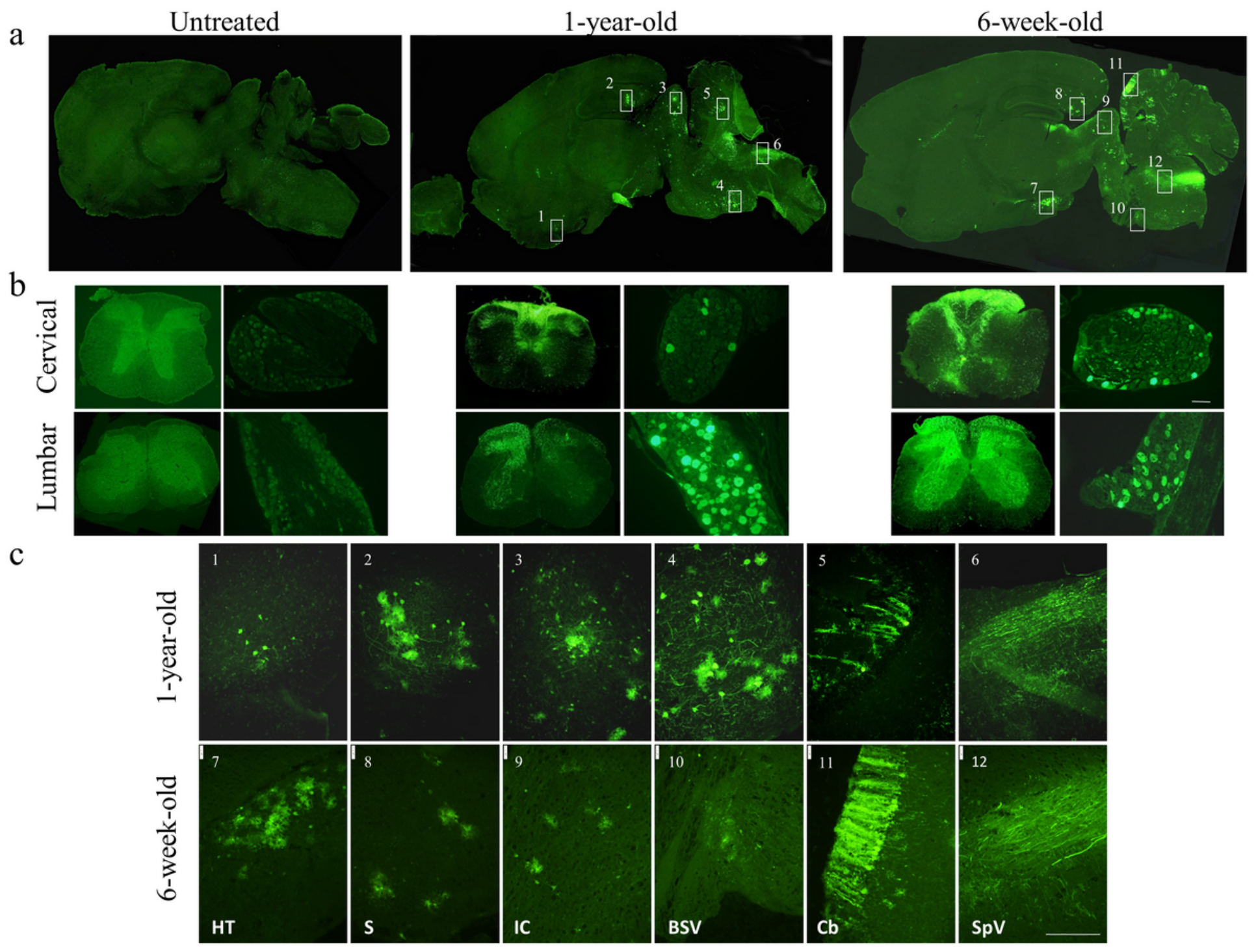

\section{Figure 1}

Expression of GFP after intrathecal injection AAV9/GFP was intrathecally injected via suboccipital puncture into 1-year-old and 6-week-old MLD mice. ( $a$ and $b$ ) Representative images of immunostaining for GFP in sagittal sections of brain (b), spinal cord and dorsal root ganglia (DRG) (b). Boxes indicate the 
approximate regions from which the higher magnification images shown in in C were acquired. (c 1 and 7 , hypothalamus (HT); 2 and 8, subiculum (S); 3 and 9, inferior colliculus (IC); 4 and 10, brain stem ventral (BSV); 5 and 11, cerebellum (Cb); 6 and 12, spinal trigeminal tract (SpV). Sections were taken 3 or 13.5 months after injection of AAV9/GFP. Bar: $200 \mu \mathrm{M}$.

a
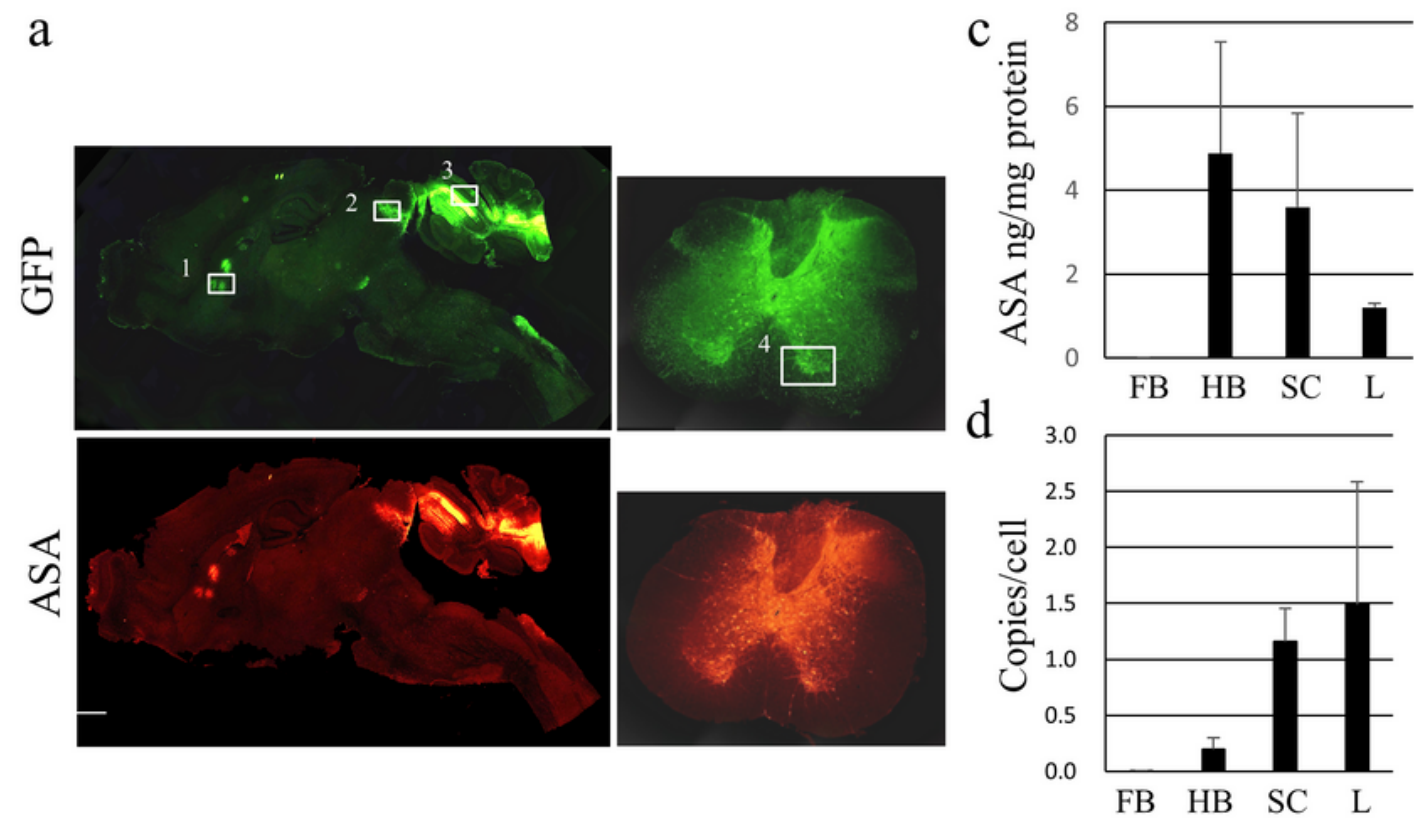

b

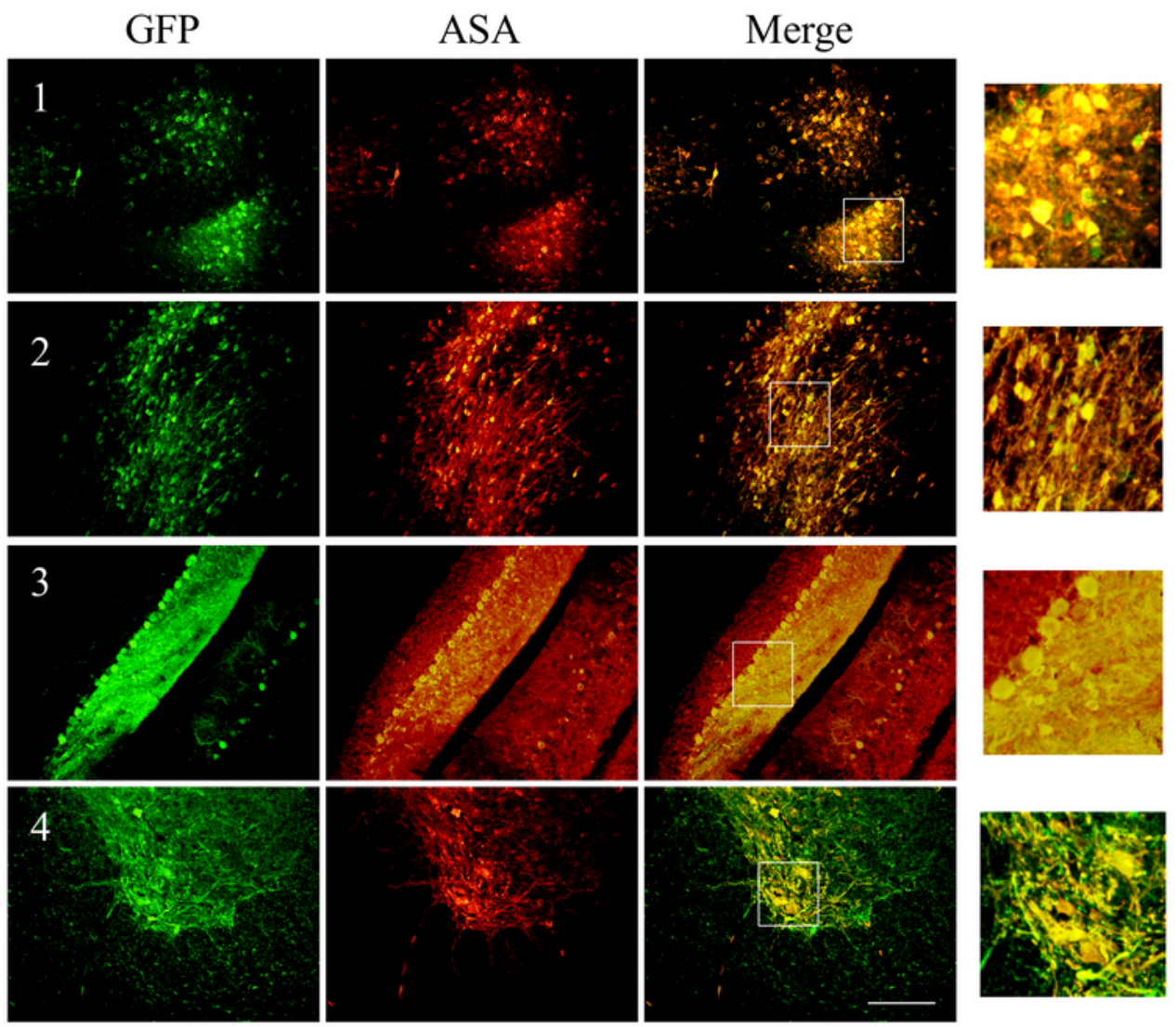

Figure 2 
Expression of GFP and ASA in the brains of treated MLD mice AAV9/ASA (encoding ASA and GFP) were intrathecally injected via suboccipital puncture into 1-year-old MLD mice. (a) representative images of immunostaining for GFP and ASA in sagittal brain and spinal cord sections taken 3 months after injection of AAV9/ASA. Boxes indicate the approximate regions from which the higher magnification images shown in B were acquired. (b) 1, caudate putamen (striatum); 2, inferior colliculus; 3 , cerebellum; 4, ventral horn of the cervical spinal cord. Bar: $200 \mu \mathrm{M}$. Panels on the right highlight the observed colocalization. (c) ASA levels in the indicated brain regions measured with ELISAs in MLD mice treated an 1 year of age $(n=5)$. (d) droplet digital PCR to detect the copy numbers of AAV vector $(n=5)$. FB, forebrain; $H B$, hindbrain; SC, spinal cord; L, liver; ASA, human arylsulfatase A. 

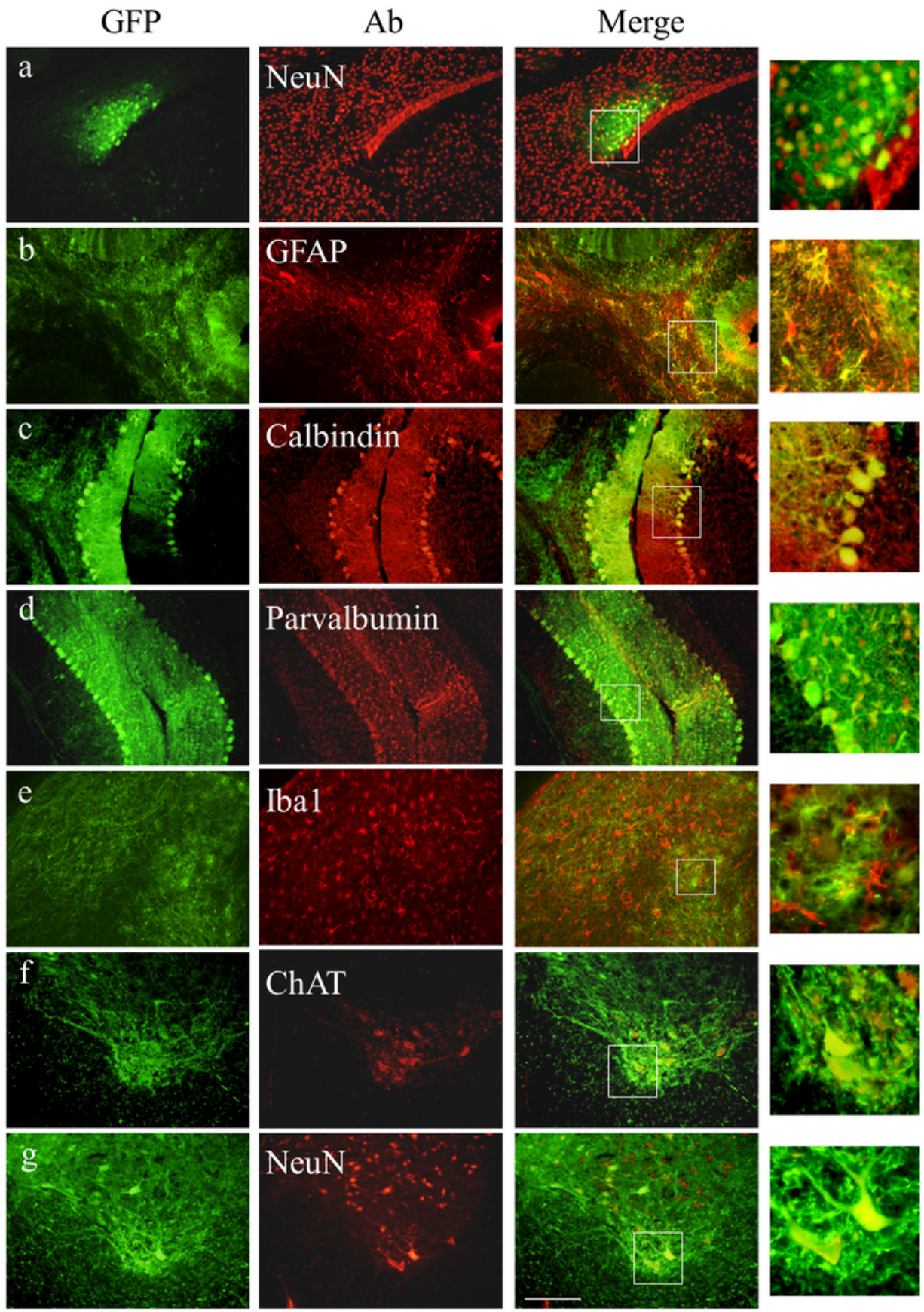

\section{Figure 3}

Immunostaining for GFP and the neuronal marker NeuN in the striatum (a), the astrocyte marker GFAP (b), the Purkinje marker calbindin (c), the basket marker parvalbumin in the cerebellum (d), the microglia marker Iba1 (e) in the brain, and ChAT-positive motor neurons (f) and NeuN-positive neurons (g) in the cervical spinal cord, especially the ventral horn. Bar: $200 \mu \mathrm{M}$. Panels on the right highlight the observed co-localization. 
$\mathrm{a}$
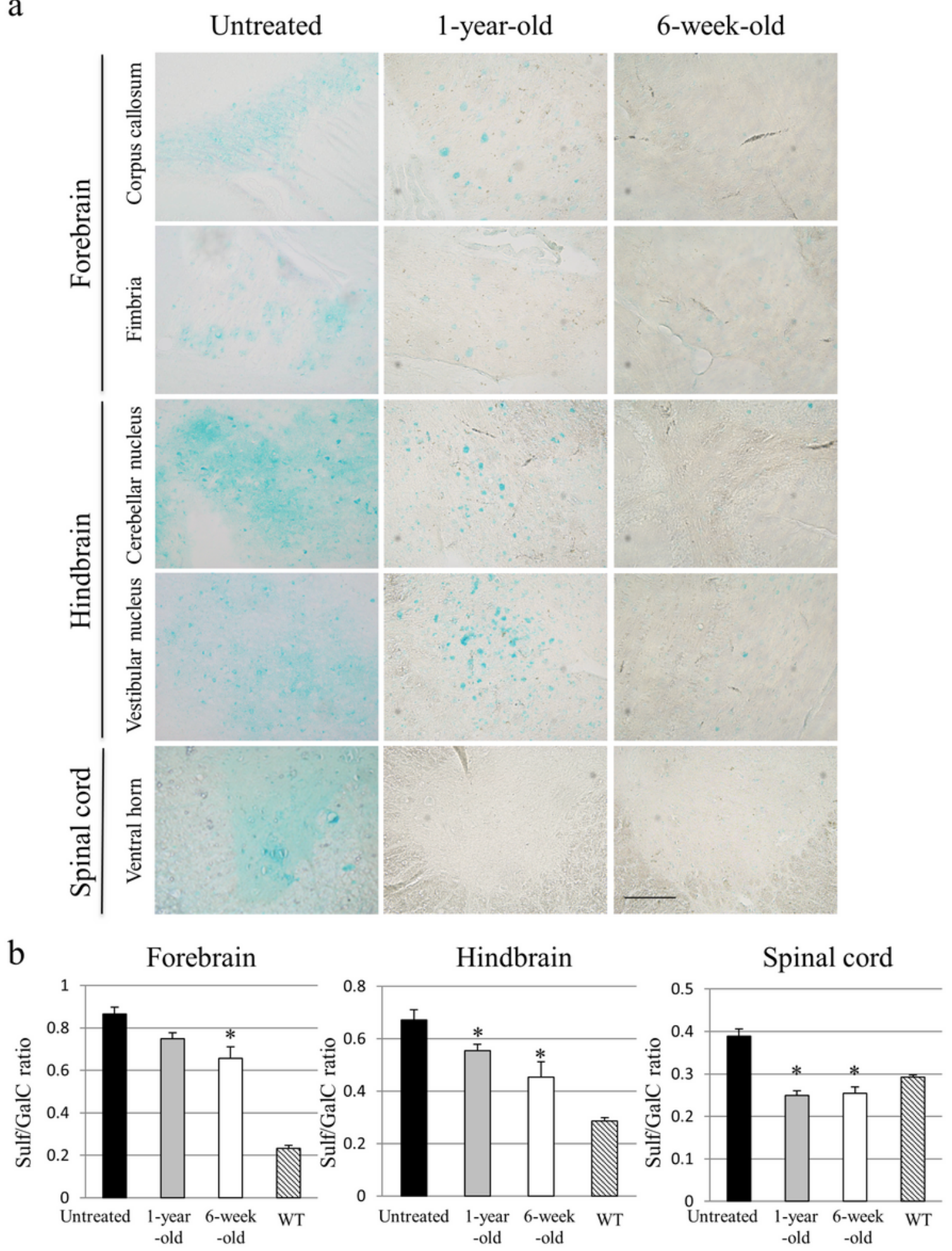

Figure 4

Correction of sulfatide storage in the brain and spinal cord (a) Alcian blue staining of sulfatide in the brain and the spinal cord of 15-month-old MLD mice left untreated or treated with AAV9/ASA at 1 year or 6 weeks of age. Bar: $200 \mu \mathrm{M}$. (b) Sulfatide content detected using thin layer chromatography. Ratios of sulfatide (Sulf) to galactosylceramide (GalC) content (Sulf/GalC ratio) in the brain and cervical spinal cord of MLD mice left untreated (closed bars, $n=5$ ), MLD mice treated at 1 year (gray bars, $n=5$ ) or 6 
weeks of age (open bars, $n=4$ ), and wild-type (WT) mice (diagonally hatched bars, $n=8$ ). * $P<0.05$ vs. untreated MLD mice.

$\mathrm{a}$
Untreated
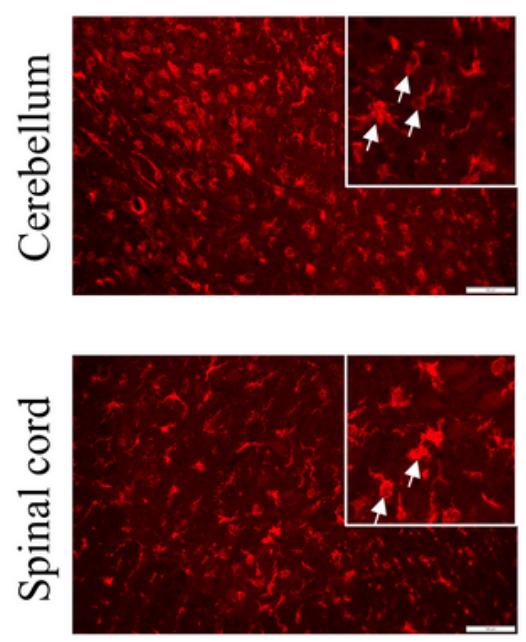

1-year-old
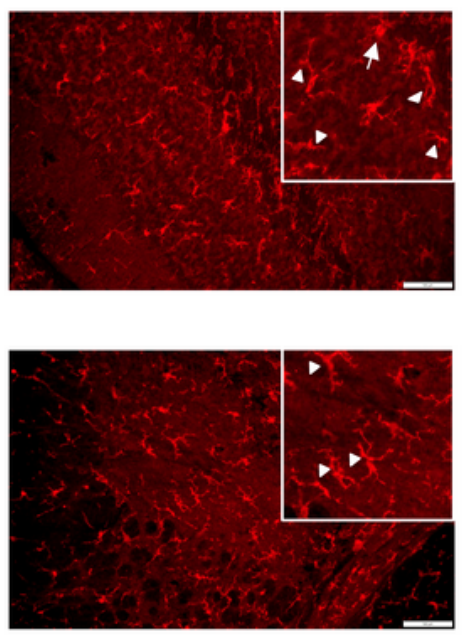

6-week-old
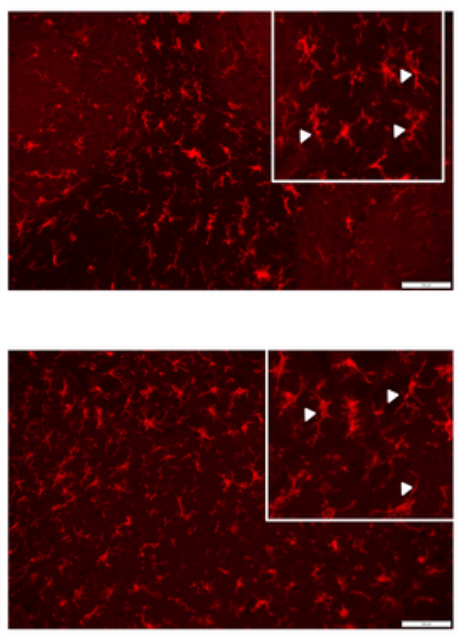

$\mathrm{b}$

Cerebellum

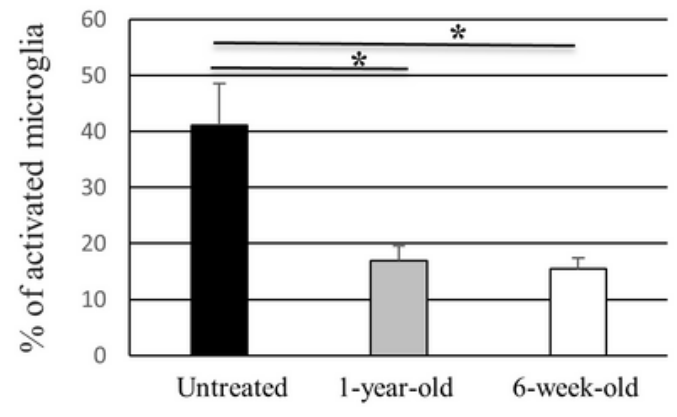

Spinal cord

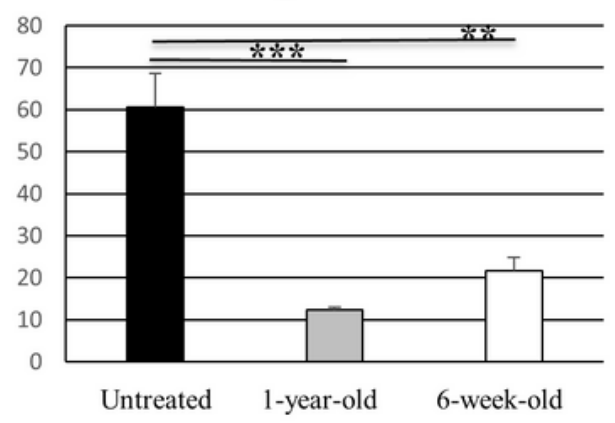

\section{Figure 5}

Reduction of activated microglia in AAV9/ASA-treated MLD mice (a) Immunostaining for the microglia marker Iba1 in the cerebellum and spinal cord. (b) Fifteen-month-old untreated mice showed amoeboid microglia cells (arrows), which correspond to activated microglia. MLD mice treated with AAV9/ASA at 1 
year or 6 weeks of age showed ramified microglia (arrow heads), which correspond to non-activated microglia. \% of activated microglia was calculated as (number of activated microglia/ numbers of Iba1 positive cells). Bar: $100 \mu \mathrm{M} .{ }^{*} p<0.05,{ }^{\star \star} p<0.03,{ }^{\star \star \star} p<0.001$ vs. untreated MLD mice.

a

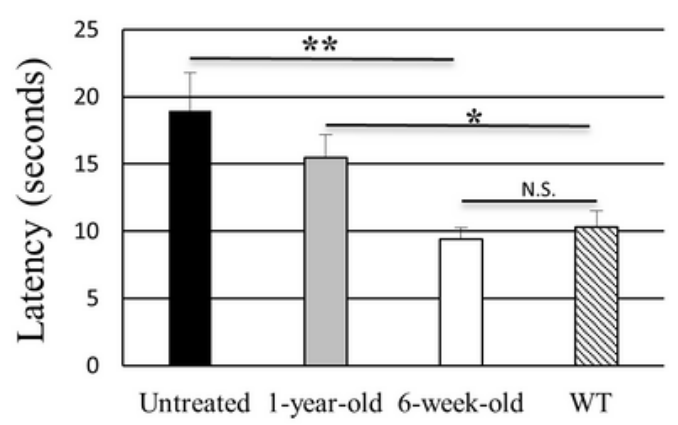

b

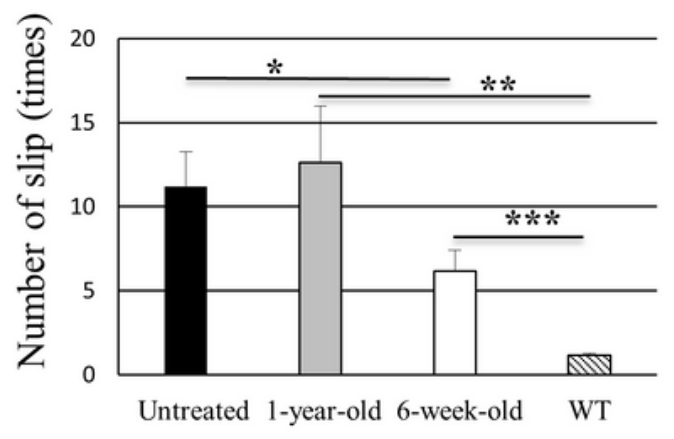

Figure 6

Therapeutic effects of intrathecal injection Motor function was assessed using a balance beam test with 15-month-old MLD mice left untreated (closed bars, $n=6$ ) or treated with AAV9/ASA at 1 year (gray bars, 
$n=5$ ) or 6 weeks of age (open bars, $n=4$ ). (a) Latency: the time required to traverse each beam. (b) Slips: the number of times the hind feet of mice slipped off a beam. All animals were given three trials on the beams. ${ }^{*} p<0.05,{ }^{*} p<0.03,{ }^{* \star *} p<0.001$ vs. untreated MLD or wild type (WT) (diagonally hatched bars, $n=8$ ) mice. 